\title{
Artery Biomineralization and its Dissolution
}

\author{
Maciej Pawlikowski \\ AGH - Univ. of Science and Technology, Dept. Mineralogy, Petrography and Geochemistry, al. Mickiewicza 30, 30-059 Cracow, Poland. \\ Corresponding Author: Maciej Pawlikowski, AGH - Univ. of Science and Technology, Dept. Mineralogy, Petrography and \\ Geochemistry, al. Mickiewicza 30, 30-059 Cracow, Poland. \\ Received date: January 27, 2020; Accepted date: February 26, 2020; Published date: March 04, 2020 Citation: Pawlikowski \\ M. (2020) Artery Biomineralization and its Dissolution. J Clinical Cardiology and Cardiovascular Interventions, 3(4); \\ DOI:10.31579/2641-0419/048
}

Copyright: (C) 2020 Maciej Pawlikowski, This is an open-access article distributed under the terms of The Creative Commons Attribution License, which permits unrestricted use, distribution, and reproduction in any medium, provided the original author and source are credited.

\begin{abstract}
This publication presents the results of studies on biomineralization of "atherosclerotic plaque" forming in the coronary arteries of the heart. Obtained results were compared to studies of an artery unaffected by the process of atherosclerosis. Different types of artery biomineralization were marked, in terms of both mineral and chemical composition. Theories on the formation of crystallization centers where "atherosclerotic plaque" develops were also presented.

Results of preliminary in vitro studies on dissolving cholesterol and phosphate mineralization in arteries were presented. The results were interpreted, and conclusions were offered.

Key Words: artery biomineralization; coronary artery; blood vessel
\end{abstract}

\section{Introduction}

The so-called atherosclerotic plaque is the cause of many health problems. Its presence in arteries often leads to death. In this work, we present results of studies on biomineralization in coronary arteries which were conducted using, among others, classical methods of mineralogy. Types and structures of the developing mineral clusters were identified, as well as their mineral and chemical composition. Details concerning the development of artery biomineralization were presented, along with preliminary results of studies on its dissolution in vitro.

Presented results may be the basis for further research on this issue, which is vitally important for human health and life.

\section{Methods and materials}

Histological, mineralogical and chemical tests of the biomineralization of coronary artery walls in the heart were conducted. Results of attempts to dissolve artery biomineralization in vitro were presented. Samples used in this study were obtained from deceased people at the John Paul II Hospital in Cracow. Due to reduction of the article's length for publication, results of studies on 5 samples were chosen (Photo 1).

List of the samples for mineralogical and chemical analysis:

\begin{tabular}{|c|c|c|}
\hline Sample & Number & Characteristics \\
\hline A & Comparative material & \\
\hline B & 124368 & fragment of coronary artery with atherosclerosis (age 63) \\
\hline C & 124911 & coronary artery with changes in its wall (age 53) \\
\hline D & 125426 & small blood vessel with atherosclerosis (age 49) \\
\hline E & 125818 & small fragment of coronary artery with atherosclerotic changes \\
\hline
\end{tabular}

The goal of the study was to recognize the method of biomineralization in the wall and intima of the coronary arteries, both in cross-section, longitudinal section along the wall, as well as changes forming on the inner wall - intima. Therefore,

The studies were conducted using a polarizing and biological microscope, as well as Geol 540 scanning microscope. EDS chemical analyses were also conducted, using an attachment for the scanning microscope. Special attention was paid to the chemical composition of the soft tissue of arterial before starting the analysis, the obtained fragments of arteries were cut in a way that made it possible to find the places where biomineralization was the strongest.

walls and mineral concentrations in the walls. Observed phenomena were documented with photos and graphs. 
The results were compared and ordered by the archive data numbers. They were discussed and interpreted in the summary. Due to reduction of this publication's length, its content was limited to consist mainly of documentation regarding the observed phenomena.

\section{Results of the analysis}

\section{Studies of arterial biomineralization}

\section{Analysis using digital binocular microscope}

Initial observations allowed us to choose five samples for analysis from the available material (Photo 1). Sample A represents an artery without atherosclerotic process. Samples B, C, D and E are coronary arteries with varying levels of atherosclerotic plaque development, obtained from people of different age.
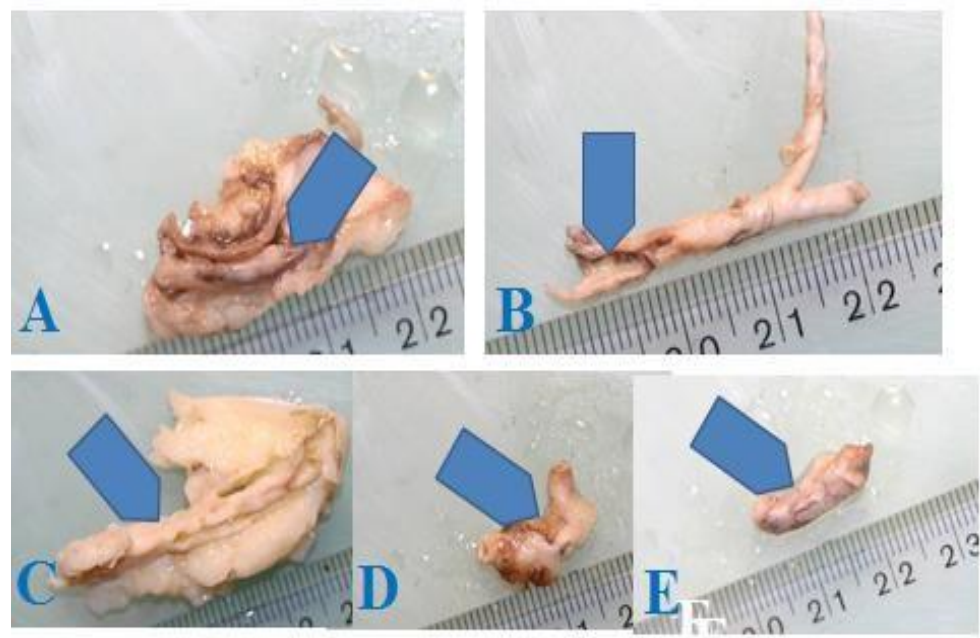

\section{Photo 1: Fragments of coronary arteries (A-E - arrows) studied for biomineralization}

\section{Studies of arterial walls using polarizing microscope}

Observations of the areas in arteries chosen for analysis were carried out using a polarizing microscope equipped with a digital camera, in partially polarized light. The microscope was used to document biomineralization phenomena (in different magnifications) and to choose samples for further studies. Preliminary observations were conducted in low magnifications, which increased as the studies progressed (Photo 2, 3).

Forms of mineralization (both organic and inorganic) were observed and compared to the artery unaffected by those processes. Materials selected at this stage were further used for histological, scanning, chemical and other analyses.

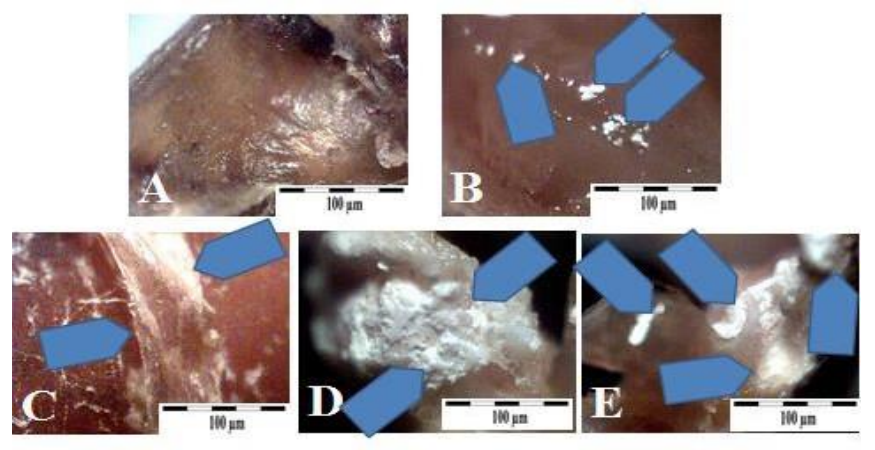

Photo 2: Microscopic images of morphology inside the walls of examined arteries, seen here in lower magnification. Sample A - young artery not affected by calcification. Samples B-E - arteries with different levels of mineralization. Digital microscope. 


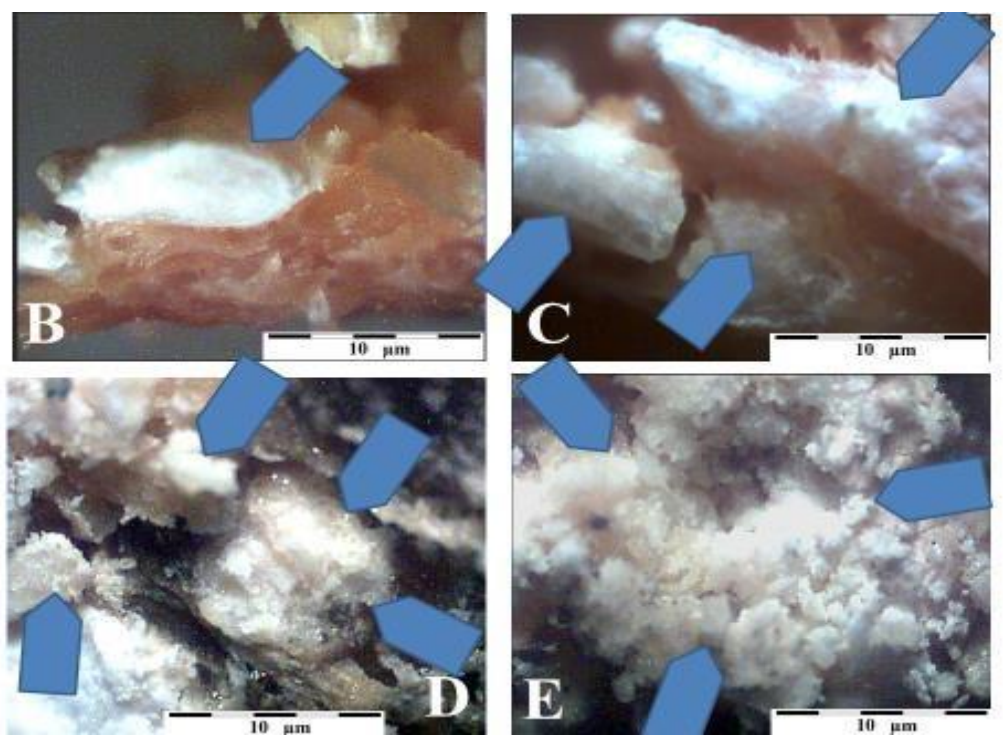

Photo 3: Cholesterol-apatite micrograins (blue arrows) in the walls of coronary arteries B, C, D, E, observed in higher magnification. Digital microscope.

\section{Studies using biological microscope (histological)}

Histological studies were conducted on preparations stained with hematoxylin. Below are the micrographs of selected preparations that offer the most information related to the studied issue. These studies allowed us to determine several main types of arterial wall biomineralization (Photo 4, 5, 6, 7, 8), which for obvious reasons were impossible to observe in the arteries themselves.
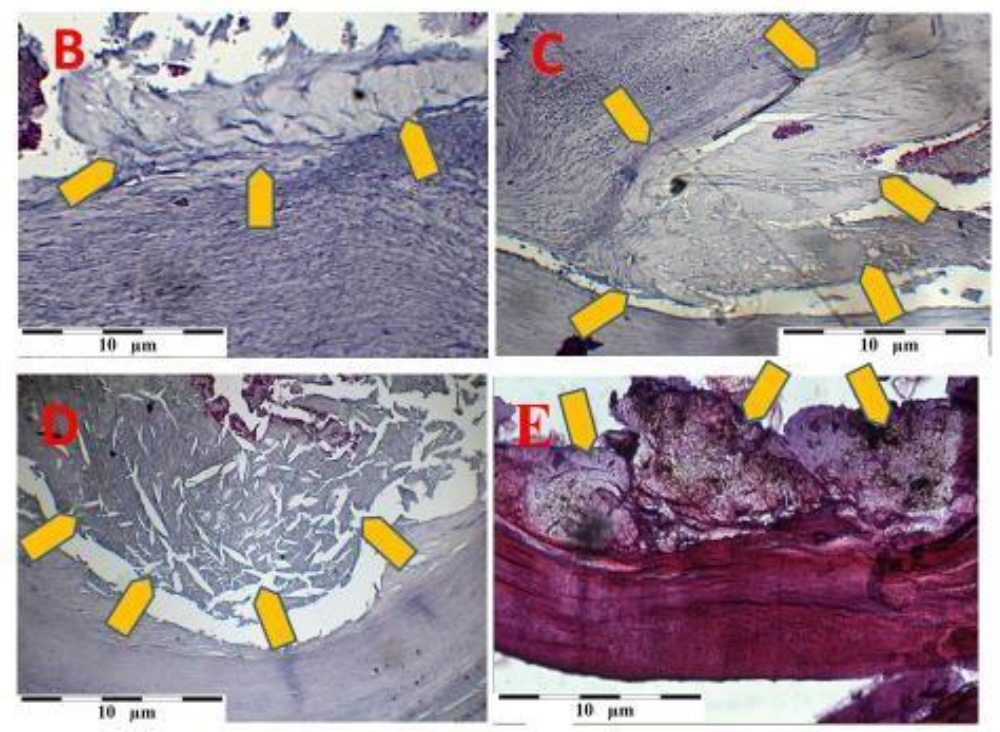

Photo 4: Types of biomineralization in coronary artery walls. B - aggregate biomineralization. C - layered biomineralization. D - recrystallizing biomineralization. $\mathrm{E}$ - crumb mineralization. Arrows indicate placement of cholesterol-mineral concentrations. Histological preparations. Biological microscope. 

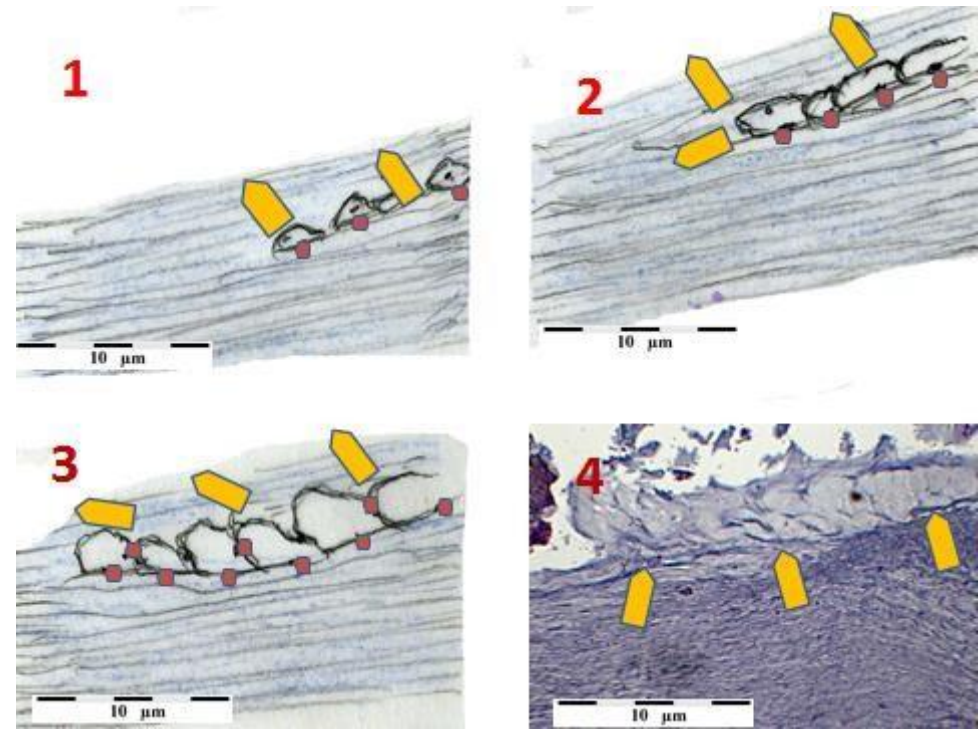

Photo 4B: Reconstruction of aggregate biomineralization. 1-4 - stages of cholesterol clusters' development. Red dots are crystallization centers where the development of cholesterol clusters started. Yellow arrows show the evolution of directions of growth in the aggregate. 4B4 - microscopic photo of aggregate biomineralization (arrows). Histological preparation. Biological microscope.
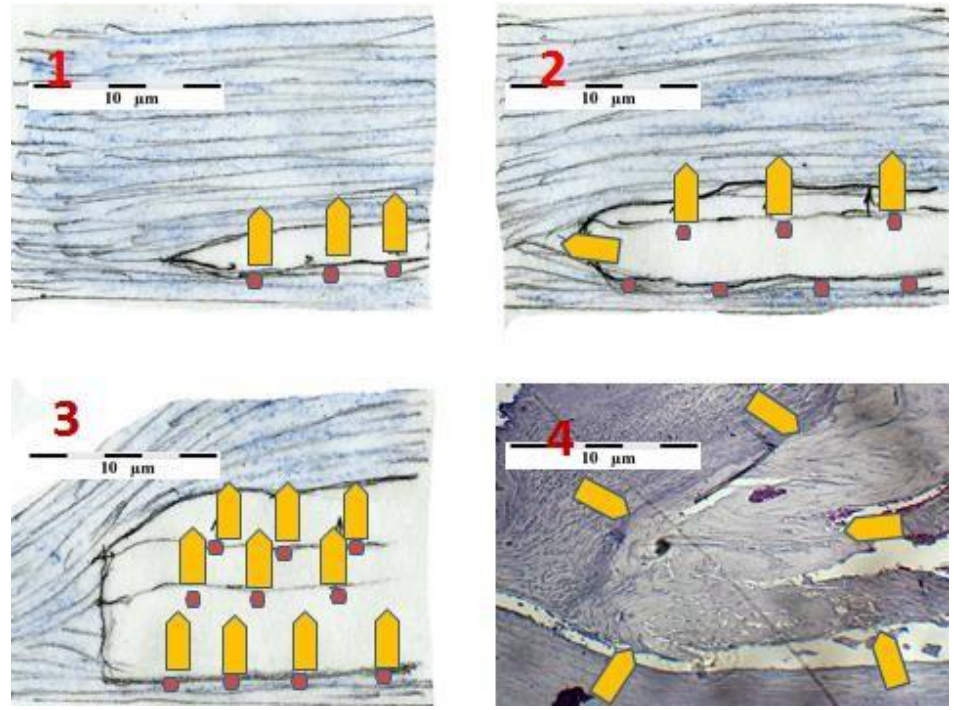

Photo 4C: Reconstruction of layered biomineralization. 1-4 - stages of development of cholesterol clusters. Red dots are crystallization centers where the crystallization of cholesterol started (they appear both in the muscle and within the already crystallized cholesterol). Yellow arrows show the evolution of directions of growth in the cholesterol cluster. 4C4 - microscopic photo of layered biomineralization (arrows). Histological preparation. Biological microscope. 

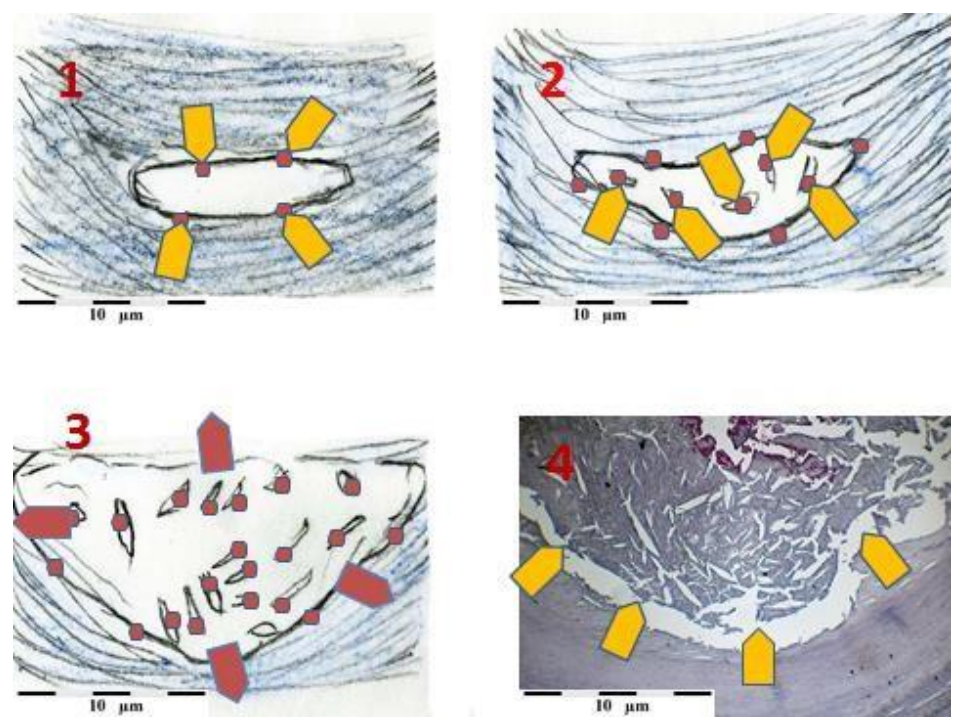

Photo 4D: Reconstruction of recrystallizing biomineralization. 1-4-stages of development of cholesterol clusters. Red dots are crystallization centers where the crystallization of cholesterol started (they appear both in the muscle and within the already crystallized cholesterol). Yellow arrows show the evolution of directions of growth in the cluster. 1 - weakly crystallized cholesterol or non-crystallized fatty substances build up. 2 - the cluster grows and the cholesterol structure organizes. Fine cholesterol crystals appear in the cholesterol cluster. 3 - further development of the cholesterol cluster and crystallization of the boat-shaped cholesterol crystals. 4D4 - microscopic photo of recrystallizing biomineralization (arrows). Histological preparation. Biological microscope.
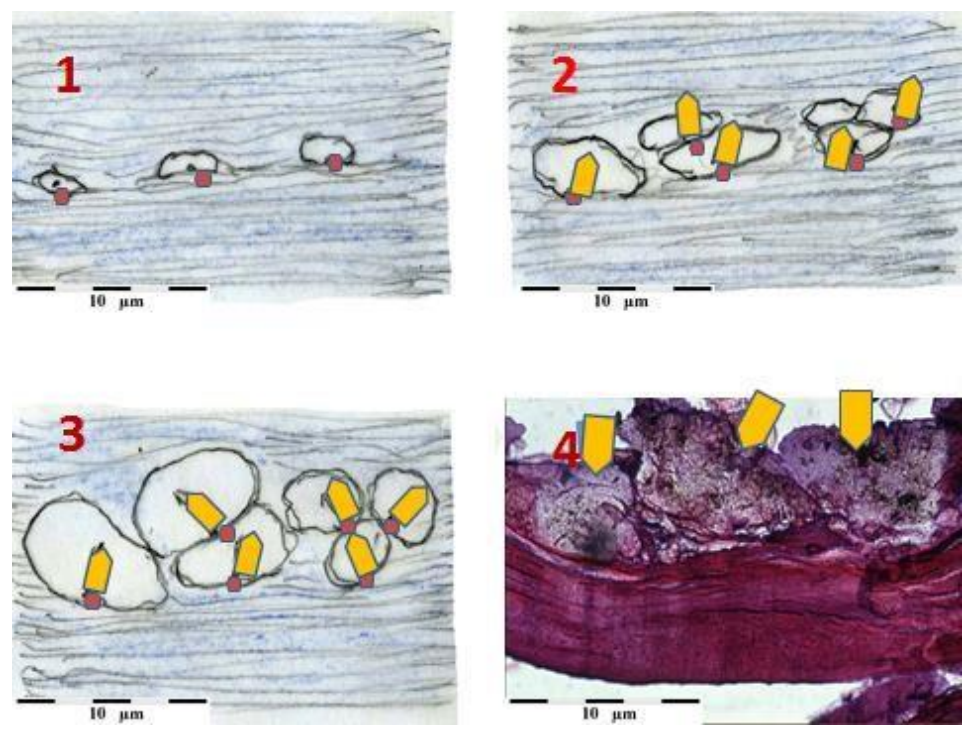

Photo 4E: Reconstruction of crumb biomineralization. 1 - beginning stage of cholesterol clusters forming in areas of muscle damage (red dots crystallization centers, yellow arrows - directions of growth in the cluster). 2 - cholesterol cluster grows, and new clusters appear. 3 - further development of the cholesterol cluster with partial change of direction of growth. 4E4 - microscopic photo of cholesterol biomineralization in arterial wall (yellow arrows). Histological preparation. Biological microscope.

Studies of mineral clusters in arterial wall using scanning microscope with attachment for chemical analysis (EDS)

EDS chemical analyses of the types of biomineralization above proved very interesting. They showed elevated levels of calcium in spaces between cholesterol clusters, as well as in some clusters themselves (Figure. 1, Photo 4E5). 


\section{CaKa, 61}

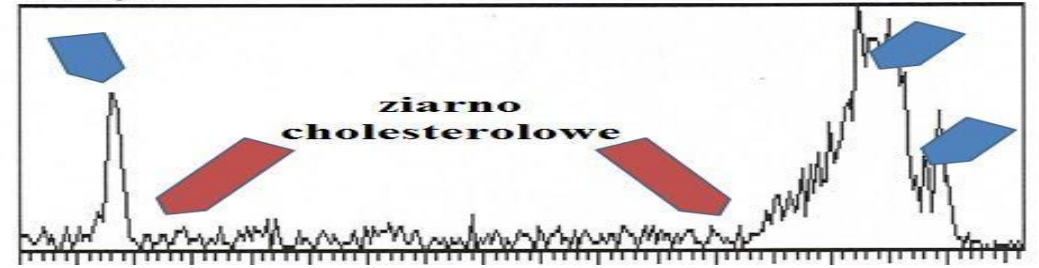

Figure 1: Sample E. Chemical analysis graph for grain mineralization. Elevated levels of calcium (blue arrows) at the edge of cholesterol grain (red arrows). SEM, linear measurement. Scale - micrometers.
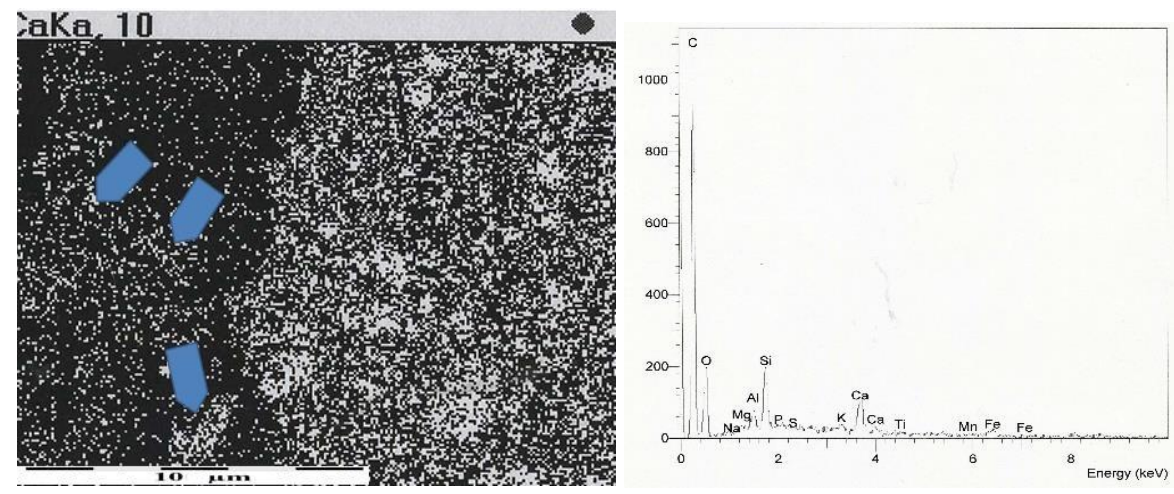

A

B

Photo 4E5: Sample E. A - contact between cholesterol grain (left, dark side of the photo). Arrows indicate elevated calcium levels within the cholesterol grain. B-EDS energy spectrum of areas with slightly elevated calcium level within cholesterol grain (A).

Studies of the intima surface using scanning microscope with an attachment for chemical analysis (EDS)

\section{Organic concentrations}

Organic concentrations forming on endothelium may be amorphous, in which case they take the form of infiltration (Photo 9A). They can also take crystalline forms. Cholesterol can form crystals. It builds aggregates or separate crystals. Such crystals develop in the spots where the inner wall of the artery has been damaged in some way (Photo 5). The crystals' structure is inhomogeneous. Next to areas that are purely cholesterol we find spots with elevated calcium levels, as indicated by EDS chemical analyses (Photo 5, 4, 5B).
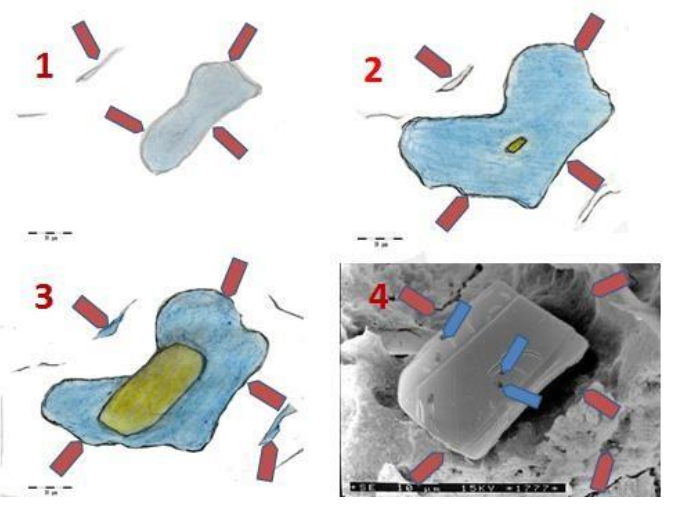

A 


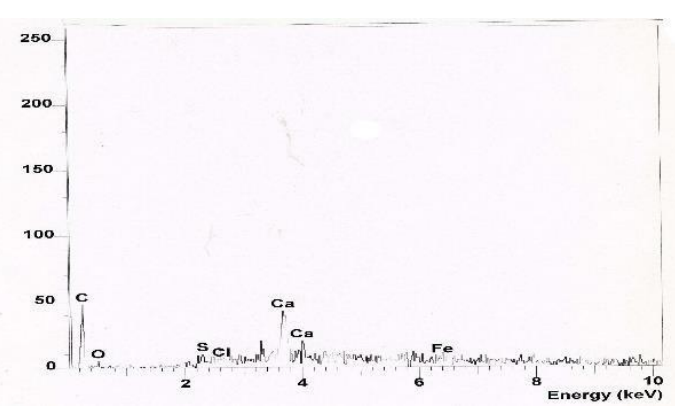

B

Photo 5: Sample B. 1-4 - stages of developing damage to the surface of the inner wall of coronary artery (red arrows) and crystallization of a cholesterol crystal (yellow crystal). 4 - microscopic image of the cholesterol crystal in a burrow created in the surface of damaged endothelium (red arrows). Blue arrows indicate marks from the electron beam used to conduct EDS chemical analyses of the cholesterol crystal. B-EDS energy spectrum from the analysis, showing presence of minor amounts of calcium in the cholesterol crystal (from Photo 5A4).

\section{Crystallization of cholesterol}

Cholesterol and cholesterol-fat concentrations develop in places where the atomic structures of the artery elements were damaged. That relates to both intima, the arterial wall, as well as its deeper parts (Photo 6).

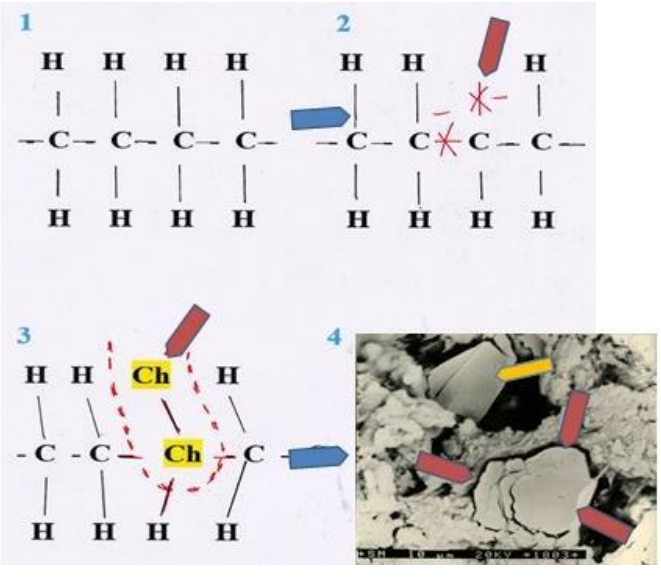

Photo 6: Reconstruction of the evolution of biological structure in the arterial muscle and development of the process of its cholesterol biomineralization. 1 - fragment of structure of the arterial muscle $(\mathrm{C}$ - carbon, $\mathrm{H}$ - hydrogen), 2 - a place where the structure was damaged. Tearing of interatomic bonds leads to development of electric field in the damaged spot. 3 - connecting cholesterol particles (arrow - Ch) and deformation of the atomic structure. Beginning of the formation of a deposit (dotted line). 4 -Sample C. Microdeposits of cholesterol in the form of a grain (red arrows) and the cholesterol crystal (yellow arrow) created in the area of damaged muscle structures. SEM.

\section{Inorganic clusters}

Those were also recognized in the walls and on the intima of arteries. The initial form of these accumulations - mostly calcium phosphates at different level of crystallinity - is a type of thin, almost invisible film on the intima. It is harmful as it doesn't allow the hormones (prostacyclin, prostaglandins and thromboxanes) to be released from the endothelium to blood.

Different stages of endothelium mineralization, from early to welldeveloped, can be seen in a single artery (Photo 7E). Phosphates often coexist with cholesterol clusters, and sometimes also with fats. 


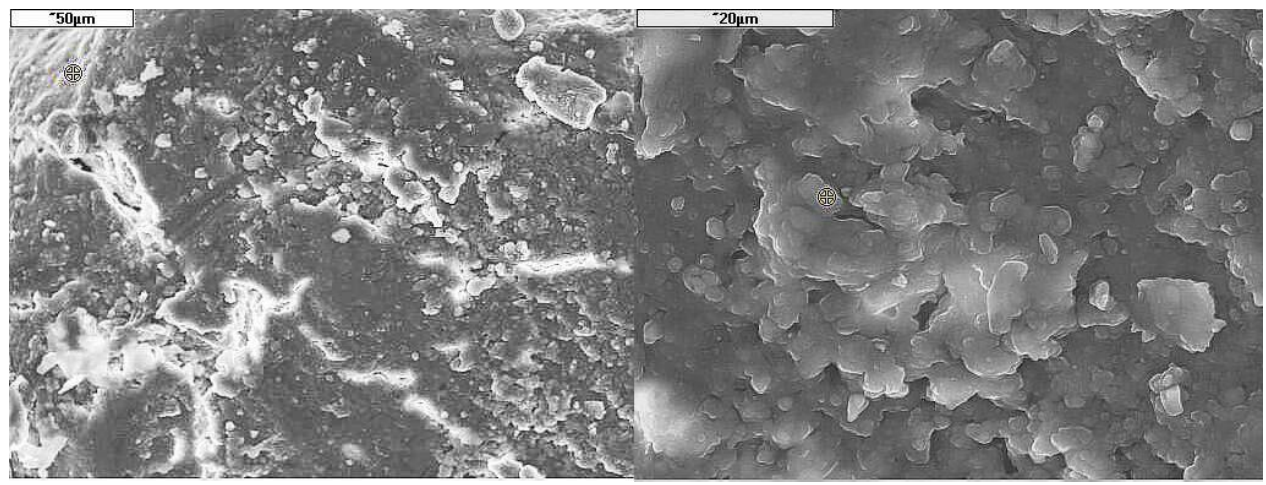

A

B

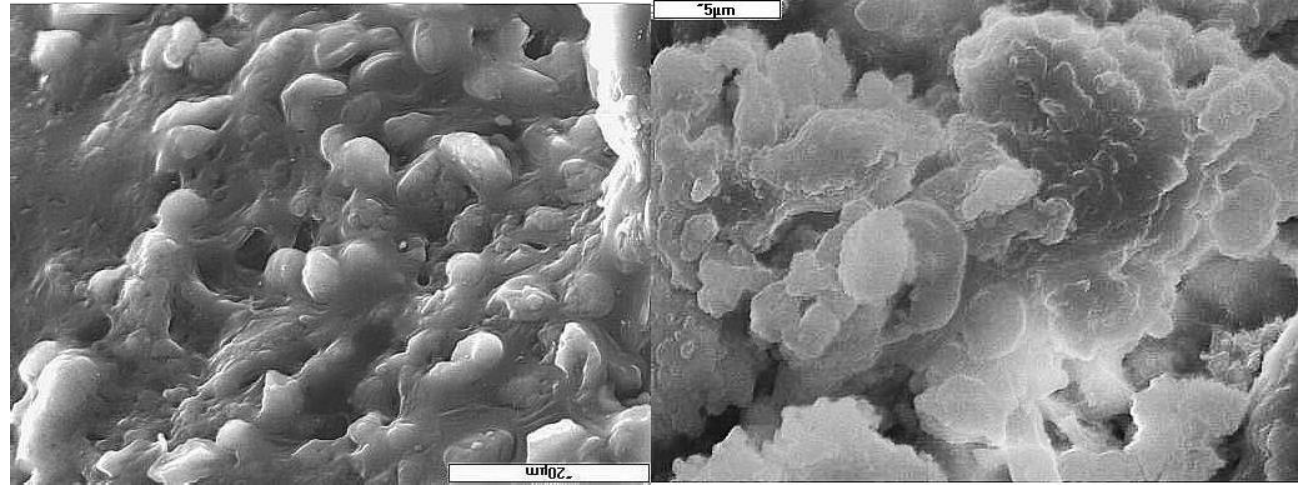

C

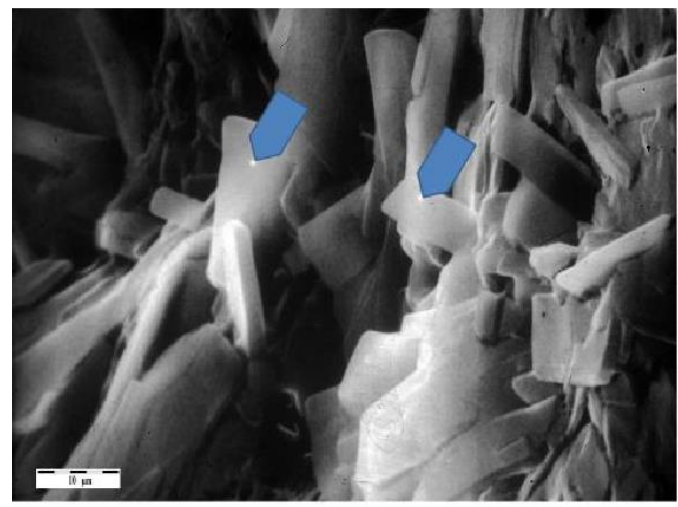

E
D

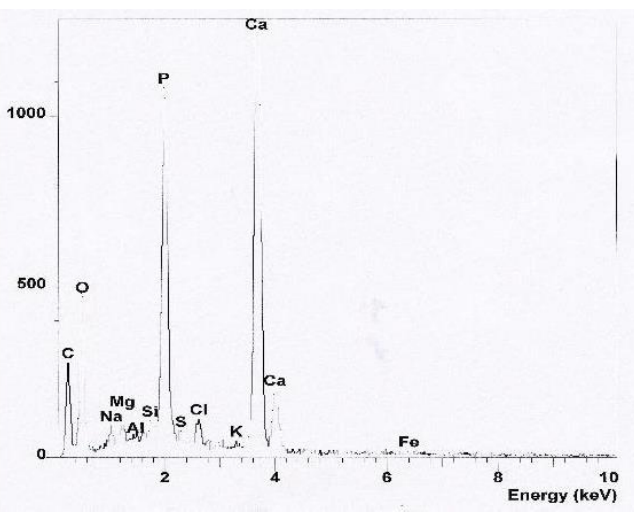

F

Photo 7: A - sample B - image of the surface of arterial endothelium covered by super thin phosphate film. B, C, D - surface of endothelium of studied arteries under different magnification, from low to high (see scale). SEM. In photos C and D, initial stages of phosphate crystallization on endothelium are visible. E - Calcium phosphates crystallizing on the surface of arterial endothelium (sample B). SEM. Arrows indicate white marks left by the electron beam used for the crystals' chemical analyses. F - EDS energy spectrum of a calcium phosphate crystal from the arterial wall's inner surface (Photo 6E).

\section{Crystallization of phosphates}

Just like crystallization of cholesterol, formation of phosphate deposits starts in the places where biological structures of the tissues are damaged
(Photo 8). Whether it is cholesterol or phosphates that crystallize in any given spot depends on what is present in the biological fluids near the damage. If calcium and phosphorus ions and cholesterol are all present in the fluids, they are all included in developing mineral cluster. 


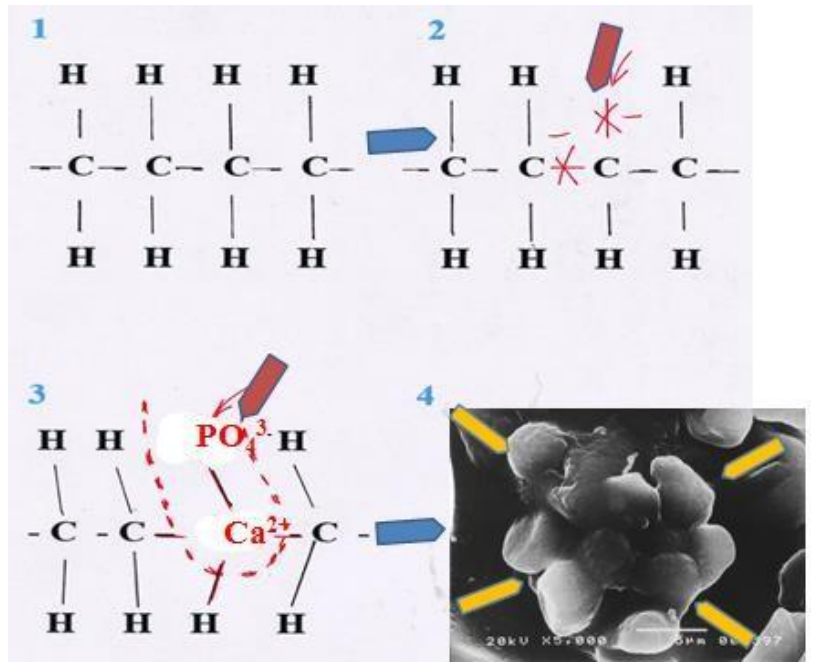

Photo 8: Reconstruction of the process of phosphate biomineralization forming in an artery. 1 - fragment of an arterial muscle structure (C - calcium, $\mathrm{H}$ - hydrogen), 2 - spot where the structure is damaged. Tearing of interatomic bonds leads to development of electric field in the damaged spot. 3 connecting calcium and phosphate ions (arrow) leads to deformation of the muscle's atomic structure. Beginning of formation of a deposit (dotted line). 4 - Sample D. Microscopic image of crystalline apatite forming in the artery. SEM.

\section{B. Studies of dissolution of arterial biomineralization (in vitro)}

Due to the complexity of the problem of dissolving deposits built up in arteries, the experiments were conducted separately for dissolving cholesterol and calcium phosphates.

\section{B.1 Dissolving cholesterol biomineralization}

Dissolution of cholesterol was attempted using arteries that were heavily filled with cholesterol. An example of such artery used for experimental dissolution of cholesterol deposits in vitro is shown in Photo 7A.

A fragment of the artery was connected to a system containing an organic solvent, peristaltic pump and a container where the solvent was collected after passing through the arteries.

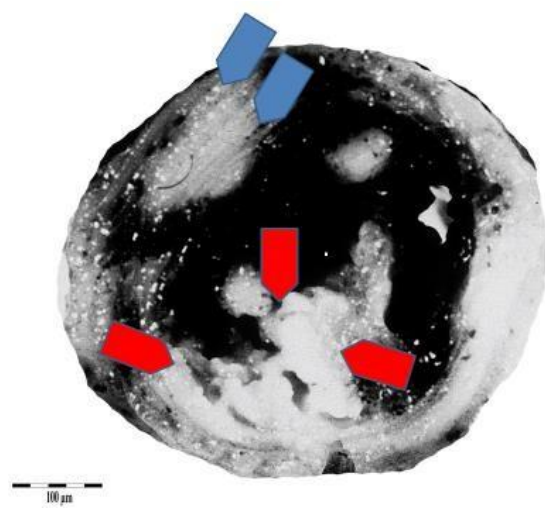

A

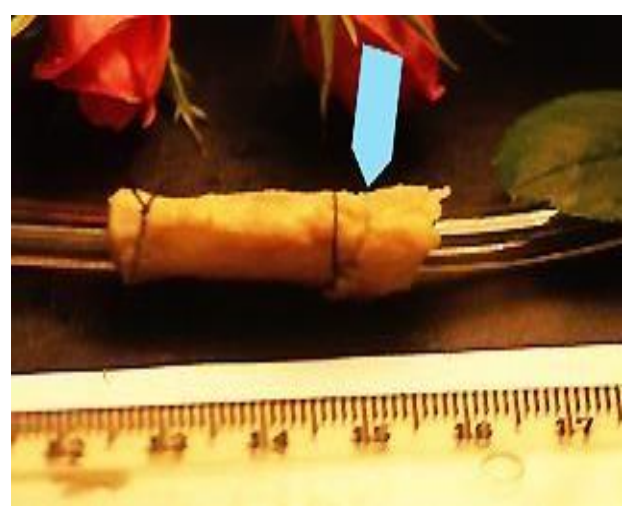

B

Photo 9: A - cross-section of an artery with two types of cholesterol clusters on the surface of the intima. Atherosclerotic plaque with signs of layering (blue arrows) and irregular cholesterol clusters in the form of crumbs, forming irregular atherosclerotic plaque (red arrows). B - artery with cholesterol deposits (arrow) connected to the experimental system for dissolution in vitro.

The organic "solvents" were evaporated after passing them through the artery, resulting in crystallization of the substances that had been dissolved in them. The crystals obtained as a result of evaporation were studied. It was determined that they were cholesterol crystals (Photo 10). 
Ethyl alcohol in an appropriate dilution proved to be the best solvent for cholesterol among those tested.

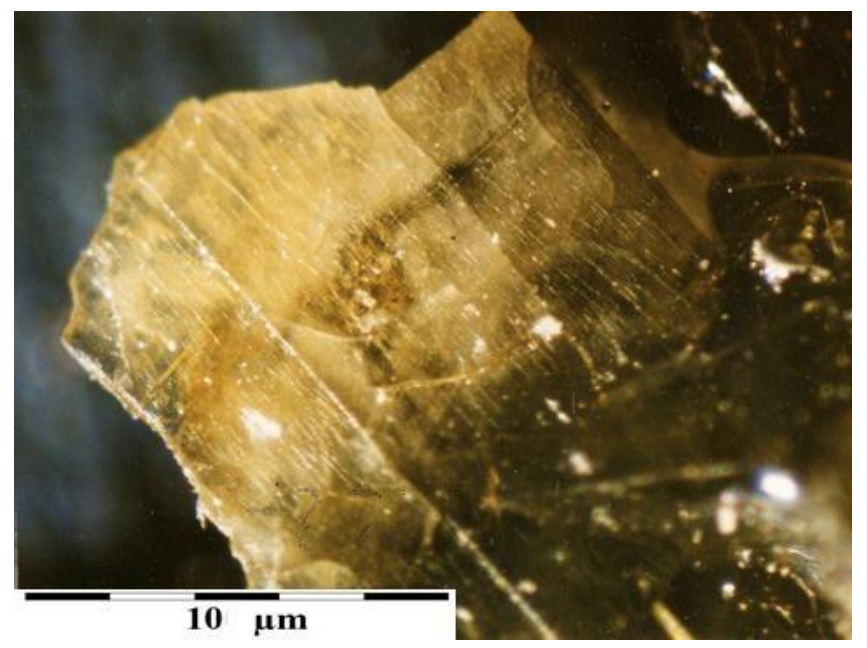

Photo 10: Cholesterol crystal in high magnification (scale), crystallized from the organic solvent passed through the arteries and then evaporated.

\section{B. 2 Dissolving phosphate biomineralization (in vitro)}

Due to trouble with obtaining arteries with high concentrations of calcifications for experiments, carbonate hydroxyapatite with a microcrystalline structure was used in the experiments focused on dissolution of phosphate biomineralization. It had been synthetized in the laboratory of the Department of Mineralogy, Petrography and Geochemistry at the AGH - Univ. of Science and Technology. Before beginning the experiments, the synthetic carbonate hydroxyapatite was analyzed mineralogically to test its structure, chemical content and crystallinity. X-ray diffractometry and EDS analyzes performed during the observation of hydroxyapatite grains using SEM were used in the studies.

Obtained synthetic was crumbled and placed in a tube used in surgery for intravenous fluid administration. The speed of the flow of "solvents" was controlled by the peristaltic pump, matching the speed of the flow to the speed of blood flow in blood vessels (Photo 11, 12). Different kinds of inorganic fluids were used as solvents. The best solvent proved to be distilled water.

Studies of the surface of carbonate hydroxyapatite grains before and after dissolution were conducted, using scanning microscope.

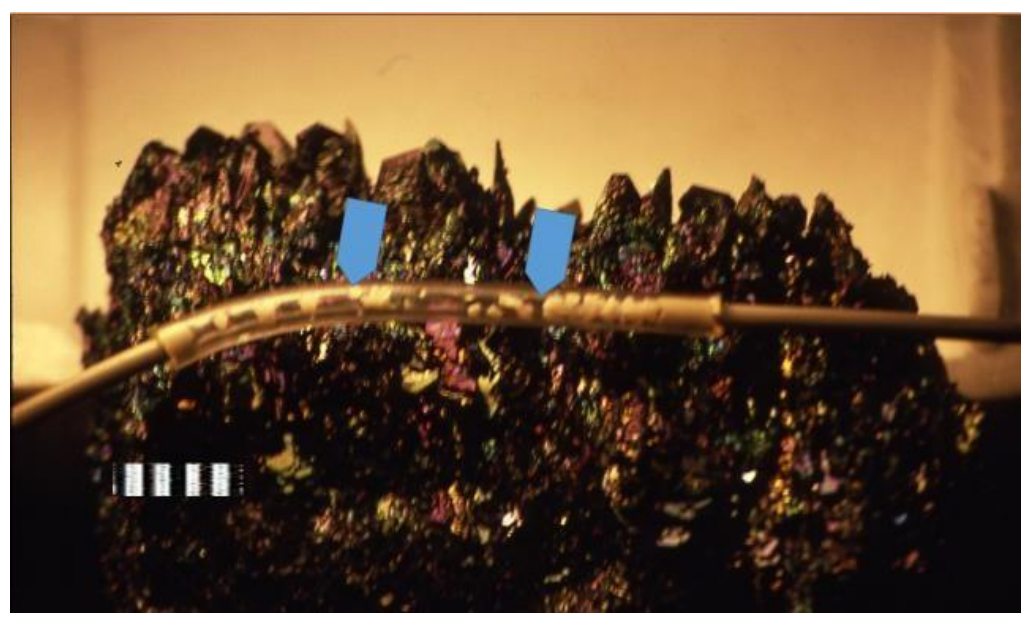

Photo 11: Tube with synthetic carbonate hydroxyapatite (arrows - material being dissolved). 


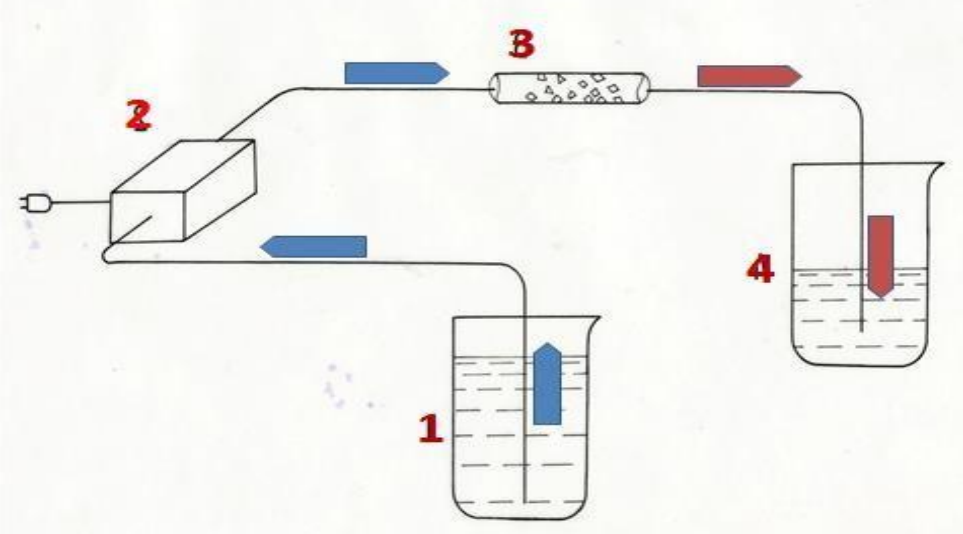

Photo 12: Diagram of a set, in which dissolution of deposits was carried out. 1 -beaker with solvent, 2 - peristaltic pump, 3 - tube with synthetic carbonate hydroxyapatite (the material being dissolved), 4 - beaker with the solvent and dissolved material after passing through the tube (3), blue and red arrows - direction of the solvent flow.

The effect of solvents on hydroxyapatite leads to the formation of micro-holes in its grains, which increase as the solvent works. The dissolved material passes into the solvent in ionic form (Photo 13).

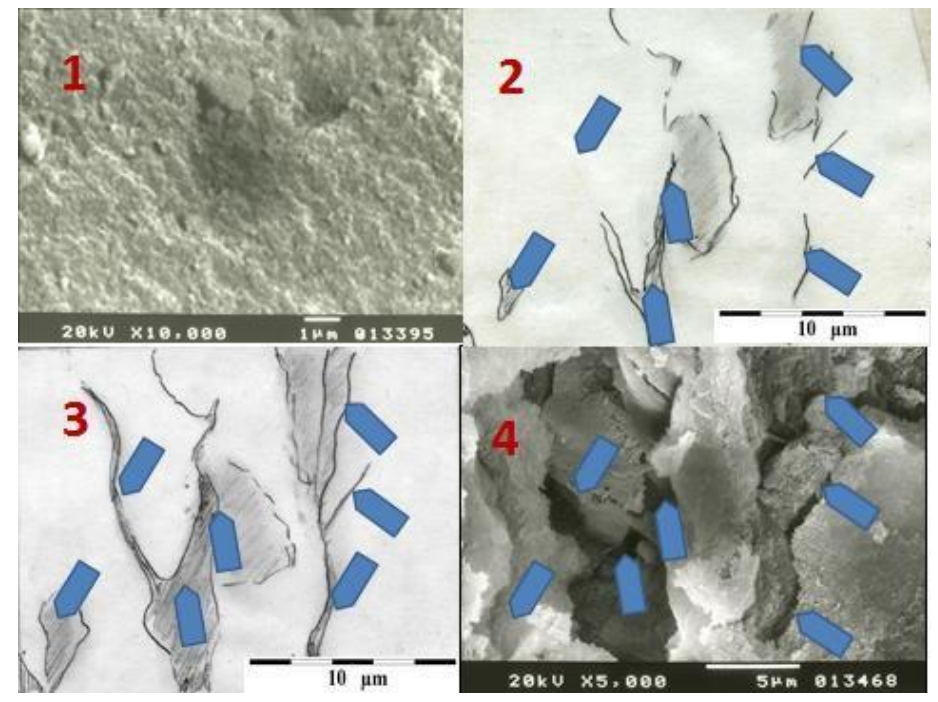

Photo 13: 1-4 - Stages of dissolution of synthetic hydroxyapatite in vitro. 1 - image (SEM) of the structure of synthetic microcrystalline carbonate hydroxyapatite used in the dissolving experiment (before dissolution). 2, 3-next stages of dissolution of the synthetic (drawings). 4 -image of the synthetic's structure (SEM) after passing through the solvent for the duration of one day. Arrows show spots with intense dissolution.

\section{Conclusions}

Studies show that biomineralization of arteries (atherosclerotic plaque) can be formed from cholesterol, phosphates or a mix of both, with varying proportions of both elements. The mixed, organic-inorganic type is the most typical.

Biomineralization (atherosclerotic plaque) forms and develops in places where the atomic structure of the artery was damaged - a place called the crystallization center. Electric field forms in the damaged spots and attracts electrically charged particles. After they attach, a deposit starts to form. The damage in the artery may occur in different places.
The reasons for the arterial damage and subsequent formation of crystallization centers vary. These could include: - genetic defects (damage),

- damage connected with physical effort and excessive mechanical "stress" on the circulatory system,

- damage caused by toxins resulting from the activity of microorganisms during an infection,

- chemical and physical factors (chemical compounds, mineral grains etc.) entering the circulatory system

- other. 
The cause of damage to the arteries (in different places) and creation of crystallization centers may be one of the above factors, a few of them, or all of the above at the same time. It is worth noting that genetic defects favor repeating the atherosclerosis due to formation of biomineralization in specific places in arteries in subsequent generations.

Substances that cause the arterial biomineralization "wander" to the crystallization centers from the fluids present in the arterial wall and from the blood transported through the artery.

Complex chemical and mineral composition of analyzed deposits proves the existence of changes in the chemical composition of the fluids from which it comes.

In addition to different crystallization of biominerals in the arterial wall and its endothelium, several methods of biomineralization formation were found in the muscles of studied arteries. Its result are deposits of different structures.

For the "atherosclerotic plaque" and arterial biomineralization to form, elevated concentration of substances that crystallize in the blood and body fluids is necessary, as well as the formation of crystallization centers. Deposits will not develop without the centers present in arteries.

Therefore, fight against arterial biomineralization (atherosclerosis) should include not just lowering the levels of crystallizing substances (cholesterol, calcium etc.), but also blocking crystallization centers.

Obtained results suggest that dissolving the deposits in arteries should be attempted using mixed, organic-inorganic "solvents". The proportion of both solvents used to fight biomineralization should be decided based on recognition of the type of biomineralization present. It should be based on mineralogical studies.

The author's earlier research proves that described phenomena of biomineralization occur also in other arteries. However, due to the extraordinary role of the heart, their presence in the coronary arteries is particularly dangerous.

Studies on dissolving arterial biomineralization should be continued using in vivo experiments.

\section{Reference}

1. Agatston A.S., Janowitz W.R., Hildner F.J., et al. (1990) Quantification of coronary artery calcium using ultrafast computed tomography. J. Am. Coll. Cardiol. ;15: 827-832.

2. Bertazzo S., Gentleman E., 2017 Aortic valve calcification: a bone of contention. Eur. Heart. J. ; 38(16): 1189-1193.

3. Bloomfield P., (2002) Choice of heart valve prosthesis. Heart ;87:583

4. Boughner D.R., Thornton M., Dunmore-Buyze J., et al. (2000) The radiographic quantitation of aortic valve calcification: implications for assessing bioprosthetic valve calcification in vitro. Physiol. Meas. ;21: 409-416.

5. Budoff M.J., Lane K.L., Bakhsheshi H., et al. (2000) Rates of progression of coronary calcium by electron beam tomography. Am. J. Cardiol. ;86: 8-11.

6. Callister T.Q., Raggi P., Cooil B., et al. (1998) Effect of HMG$\mathrm{CoA}$ reductase inhibitors on coronary artery disease as assessed by electron-beam computed tomography. N. Engl. J. Med. ;339: 1972-1978.

7. Ewe S.H., Ng A.C., Schuijf J.D., et al. (2011) Location and severity of aortic valve calcium and implications for aortic regurgitation after transcatheter aortic valve implantation. Am. J. Cardiol. ;108:1470-1477.

8. Gohlke-Barwolf C., (2000) Anticoagulation in valvar heart disease: new aspects and management during non-cardiac surgery. Heart ;86:715-721.

9. Enriquez-Sarano M ., (2002) Timing of mitral valve surgery. Heart ;87:

10. Haensig M., Lehmkuhl L., Rastan A.J., et al. (2012) Aortic valve calcium scoring is a predictor of significant paravalvular aortic insufficiency in Surg. ;41:1234-1241.

11. Harbaoui B., Montoy M., Charles P., et al. (2016) Aorta calcification burden: towards an integrative predictor of cardiac outcome after transcatheter aortic valve implantation. Atherosclerosis; 246: 161-168

12. Herzog C.A., Ma J.Z., Collins A.J., (2002) Long-term survival of dialysis patients in the United States with prosthetic heart valves. Should ACC/AHA practice guidelines on valve selection be modified? Circulation ;105:1336-1341.

13. Iivanainen A.M., Lindroos M., Tilvis R., et al. (1996) Calcific degeneration of the aortic valve in old age: is the development of flow obstruction predictable? J. Intern. Med. ;239: 269-273.

14. Kapila, R. , (1986) Hart calcific cerebral emboli and aortic stenosis: detection by computed tomography. Stroke, $17 ; 619$ 621.

15. Khetarpal V., Mahajan N., Madhavan R., Batra S., Mopala P., Sagar A., Rapolu P., Nangia S., Afonso L., (2009) Calcific aortic valve and spontaneous embolic stroke: a review of literature. J. Neurol. Sci. ;287(1-2): 32-53

16. Kizer J.R., Gefter W.B., de Lemos A.S., et al. (2001) Electron beam computed tomography for the quantification of aortic valve calcification. J. Heart Valve Dis. ;10: 361-366.

17. Kleczyński P., Dziewierz A., Daniec M., et al. (2017) Impact of post-dilatation on the reduction of paravalvular leak and mortality after transcatheter aortic valve implantation. Kardiol. Pol. ; 75: 742-748

18. Khalique O.K., Hahn R., Gada H., et al. (2014) Quantity and location of aortic valve complex calcification predicts severity and location of paravalvular regurgitation and frequency of post-dilation after balloon-expandable transcatheter aortic valve replacement. JACC Cardiovasc Interv. ; 7:885-894.

19. Koos R, Mahnken AH, Dohmen G, et al. (2011) Association of aortic valve calcification severity with the degree of aortic regurgitation after transcatheter aortic valve implantation. Int. J. Cardiol. ; 150: 142-145.

20. Lindblom D,, Lindblom U., Qvist J., et al. (2001) Long-term relative survival rates after heart valve replacement. J. Am. Coll. Cardiol. ;15:566-573.

21. Lindroos M., Kupari M., Valvanne J., et al. (1994) Factors associated with calcific aortic valve degeneration in the elderly. Eur. Heart. J. ;15: 865-870.

22. Mako W.J., Vesely I., (1997) In vivo and in vitro models of calcification in porcine aortic valve cusps. J. Heart Valve Disease ; 6(3); 316-323.

23. Massel D., Little S.H., (2001) Risks and benefits of adding antiplatelet therapy to warfarin among patients with prosthetic heart valves: a meta-analysis. J. Am. Coll. Cardiol. ;37:569-578. 
24. Mohler E.R., (2000) Are atherosclerotic processes involved in aortic-a.valve calcification? Lancet. ;356: 524-525.

25. Nagueh S., Sun H., Kopelen H., et al. (2001) Hemodynamic determinants of the mitral annulus diastolic velocities by tissue Doppler. J. Am. Coll. Cardio. 1;37:278-285.

26. Otto C.M., Lind B.K., Kitzman D.W., et al. (1999) Association of aortic-valve sclerosis with cardiovascular mortality and morbidity in the elderly. N. Engl. J. Med. ;341: 142-147.

27. Otto C.M., Kuusisto J., Reichenbach D., et al. (1994) Characterisation of the early lesion of "degenerative" valvular aortic stenosis: histological and immunohistochemical studies. Circulation. ;90: 844-853.

28. Otto C.M., Burwash IG, Legget ME, et al. (1997) Prospective study of asymptomatic valvular aortic stenosis: clinical, echocardiographic, and exercise predictors of outcome. Circulation. ;95: 2262-2270.

29. Pibarot P., Dumesnil J., (2000) Hemodynamic and clinical impact of prosthesis-patient mismatch in the aortic valve position and its prevention. J. Am. Coll. Cardiol. ;36:11311141.

30. Peter M., Hoffmann A., Parker C., et al. (1993) Progression of aortic stenosis: role of age and concomitant coronary artery disease. Chest ;103: 1715-1719.

31. R. Pfitzner R., et al. (2018) Influence of cryopreservation on structural, chemical and immunoenzymatic properties of the aortic valve allografts. Transplantation Proceedings, 2018, 50, (7), 2195-2198
Piper C., Körfer R., Horstkotte D., (2000) Prosthetic valve

33. Rumberger J.A., Simons D.B., Fitzpatrick L.A., et al. (1995) Coronary artery calcium area by electron-beam computed tomography and coronary atherosclerotic plaque area: a histopathologic correlative study. Circulation. ;92: 2157-2162.

34. Ryś M., Hryniewiecki T., Michałowska I., Stokłosa P., Różewicz-Juraszek M., Chmielak Z., Dąbrowski M., Mirota K., Szymański P., (2018) Quantitative estimation of aortic valve calcification in multislice computed tomography in predicting the development of paravalvular leaks following transcatheter aortic valve replacemen. Postepy Kardiol. Interwencyjnej ; 14(1): 85-89.

35. Sangiorgi G., Rumberger J.A., Severson A., et al. 1998 Arterial calcification and not lumen stenosis is highly correlated with atherosclerotic plaque burden in humans: a histologic study of 723 coronary artery segments using nondecalcifying methodology. J. Am. Coll. Cardiol. ;31: 126-133.

36. Stewart B.F., Siscovick D., Lind B.K., et al. 1997 Clinical factors associated with calcific aortic valve disease. J. Am. Coll. Cardiol. ;29: 630-634.

37. Takamiya Y., Miura S., Sako H., Shirai K., Morishige N. , Tashiro T. , Saku K., (2007) Pseudoaneurysm of the mitralaortic intervalvular fibrosa following infective endocarditis in a patient with acute heart failure: a case report. J. Cardiol, ;49 ;353-356.

38. Pawlikowski M, Pfitzner R (1999) Mineralization of heart, (Mineralization of heart and big blood vessels). Wyd IGSMiE

PAN Kraków pp. 142

32.

This work is licensed under Creative Commons Attribution 4.0 License

To Submit Your Article Click Here: Submit Article
Ready to submit your research? Choose Auctores and benefit from:

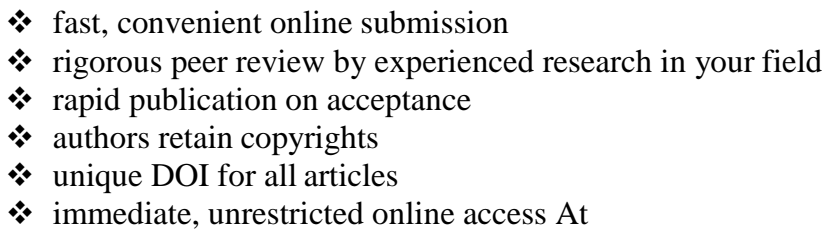

Auctores, research is always in progress.

Learn more www.auctoresonline.org/journals/clinical-cardiology-andcardiovascular-interventions 